\title{
Article
}

Subscriber access provided by Northwestern Univ. Library

\section{A new three-dimensional subsulfide IrInS with Dirac semimetal behavior}

Jason F. Khoury, Alexander J. E. Rettie, Mojammel A. Khan, Nirmal J. Ghimire, Iñigo Robredo, Jonathan E. Pfluger, Koushik Pal, Christopher Wolverton, Aitor Bergara, Jidong Samuel Jiang, Leslie M. Schoop, Maia G. Vergniory, J.F. Mitchell, Duck Young Chung, and Mercouri G. Kanatzidis J. Am. Chem. Soc., Just Accepted Manuscript • DOI: 10.1021/jacs.9b10147 • Publication Date (Web): 07 Nov 2019

Downloaded from pubs.acs.org on November 7, 2019

\section{Just Accepted}

"Just Accepted" manuscripts have been peer-reviewed and accepted for publication. They are posted online prior to technical editing, formatting for publication and author proofing. The American Chemical Society provides "Just Accepted" as a service to the research community to expedite the dissemination of scientific material as soon as possible after acceptance. "Just Accepted" manuscripts appear in full in PDF format accompanied by an HTML abstract. "Just Accepted" manuscripts have been fully peer reviewed, but should not be considered the official version of record. They are citable by the Digital Object Identifier (DOI@). "Just Accepted" is an optional service offered to authors. Therefore, the "Just Accepted" Web site may not include all articles that will be published in the journal. After a manuscript is technically edited and formatted, it will be removed from the "Just Accepted" Web site and published as an ASAP article. Note that technical editing may introduce minor changes to the manuscript text and/or graphics which could affect content, and all legal disclaimers and ethical guidelines that apply to the journal pertain. ACS cannot be held responsible for errors or consequences arising from the use of information contained in these "Just Accepted" manuscripts. 


\title{
A new three-dimensional subsulfide $\operatorname{Ir}_{2} \ln _{8} S$ with Dirac semimetal behavior
}

Jason F. Khoury ${ }^{1}$, Alexander J. E. Rettie ${ }^{2,3}$, Mojammel A. Khan ${ }^{2}$, Nirmal J. Ghimire ${ }^{2}$, Iñigo Robredo $^{4,5}$, Jonathan E. Pfluger ${ }^{6}$, Koushik Pal ${ }^{6}$, Chris Wolverton ${ }^{6}$, Aitor Bergara ${ }^{4,5,7}$, J. S. Jiang ${ }^{2}$, Leslie M. Schoop ${ }^{8}$, Maia G. Vergniory ${ }^{4,9}$, J. F. Mitchell ${ }^{2}$, Duck Young Chung ${ }^{2}$, and Mercouri G. Kanatzidis ${ }^{1,2 *}$

${ }^{1}$ Department of Chemistry, Northwestern University, Evanston, Illinois 60208, United States

${ }^{2}$ Materials Science Division, Argonne National Laboratory, Argonne, Illinois 60439, United States

${ }^{3}$ Department of Chemical Engineering, University College London, London WC1E 7JE, United Kingdom

${ }^{4}$ Donostia International Physics Center, Paseo Manuel de Lardizabal 4, 20018 Donostia-San Sebastian, Spain

${ }^{5}$ Condensed Matter Physics Department, University of the Basque Country UPV/EHU, 48080 Bilbao, Spain

${ }^{6}$ Department of Materials Science and Engineering, Northwestern University, Evanston, Illinois 60208, United States

${ }^{7}$ Centro de Física de Materiales, Centro Mixto CSIC-UPV/EHU, 20018 Donostia, Spain ${ }^{8}$ Department of Chemistry, Princeton University, Princeton, New Jersey 08540, United States ${ }^{9}$ Ikerbasque, Basque Foundation for Science, E-48011 Bilbao, Spain

\begin{abstract}
Dirac and Weyl semimetals host exotic quasiparticles with unconventional transport properties, such as high magnetoresistance and carrier mobility. Recent years have witnessed a huge number of newly predicted topological semimetals from existing databases; however, experimental verification often lags behind such predictions. Common reasons are synthetic difficulties or the stability of predicted phases. Here, we report the synthesis of the Type-II Dirac semimetal $\operatorname{Ir}_{2} \operatorname{In}_{8} S$, an air-stable compound with a new structure type. This material has two Dirac crossings in its electronic structure along the $\Gamma-Z$ direction of the Brillouin zone. We further show that $\operatorname{Ir}_{2} \operatorname{In}_{8} \mathrm{~S}$ has a high electron carrier mobility of $\sim 10,000 \mathrm{~cm}^{2} / \mathrm{Vs}$ at $1.8 \mathrm{~K}$, and a large, non-saturating transverse magnetoresistance of $\sim 6000 \%$ at $3.34 \mathrm{~K}$ in a $14 \mathrm{~T}$ applied field. Shubnikov de-Haas oscillations reveal several small Fermi pockets and the possibility of a nontrivial Berry phase. With its facile crystal growth, novel structure type, and striking electronic structure, $\operatorname{Ir}_{2} \operatorname{In}_{8} S$ introduces a new material system to study topological semimetals and enable advances in the field of topological materials.
\end{abstract}




\section{Introduction}

The field of topological semimetals (TSMs) has been blossoming since the discovery of several three-dimensional (3D) materials with exotic physical properties, such as $\mathrm{Cd}_{3} \mathrm{As}_{2}$ and $\mathrm{Na}_{3} \mathrm{Bi}^{1-7}$ As $3 \mathrm{D}$ analogues of graphene, these materials have linearly dispersing bands that cross at the Fermi level to create four-fold degenerate Dirac points in momentum space and are referred to as Dirac semimetals (DSMs). These Dirac points harbor massless Dirac fermions that engender remarkable transport properties, including high carrier mobility and extremely large magnetoresistance. ${ }^{8-11}$ If a material with symmetry protected band crossings lacks inversion or time reversal symmetry (TRS), the massless fermions are only two-fold degenerate instead of four, referred to as a Weyl semimetal (WSM). ${ }^{6,8}$,9, 12 These Weyl fermions have different surface states and transport behavior compared to DSMs due to their inherent chirality or "handedness". ${ }^{13}$ The interest in TSMs is not limited to just their transport properties; it also extends to other areas such as optical switches of infrared sensors. ${ }^{14}$ In addition, it has been predicted that topological superconductivity (TSC) could arise in type-II DSMs with tilted Dirac cones. ${ }^{15-21}$ Understanding the physical phenomena linked to DSMs and advancing the knowledge of how Dirac and Weyl fermions behave calls for new and diverse examples of stable material systems in which they reside.

To date, there exist a few dozen experimentally verified topological semimetals, while many more have been predicted but not yet synthesized. ${ }^{6-7}$ There are many factors that slow down or impede experimental verification of predictions: First, the predicted materials might not be synthesizable in single crystalline form. Second, they might be extremely air sensitive, which will drastically complicate measurements. Third, a wide combination of different experimental techniques is required to definitively determine the topological nature of a system. Lastly, 
prediction based on DFT can be incorrect, either by underestimating band gaps, omitting magnetic order, or by predicting unreliable materials. ${ }^{22}$ The currently available candidate materials are limited in their structural diversity, with most corresponding to the same handful of structure types. In particular, the class of square net materials has garnered considerable attention as a source for TSM candidates because of their wide range of tunable and synthesizable analogues. ${ }^{23}$ Still, these materials all show related electronic structures as a result of their similar crystal structures. Synthesizing air-stable TSMs with novel structure types as well as facile growth conditions is important in further understanding their properties, as there are several different types of fermion degeneracies predicted to exist that have not yet been verified experimentally. ${ }^{24-25}$ Considering recent advances that catalogued all known materials based on their topological properties, new structure types and compounds have become crucial for advancing the field. ${ }^{26-30}$ Indeed, more unique candidate materials and diverse structure types must be identified to provide new insight in understanding topological behavior. In this work, we describe the synthesis, crystal structure, and electronic transport characterization of $\operatorname{Ir}_{2} \mathrm{In}_{8} \mathrm{~S}$, a new, air-stable subsulfide compound that represents a Dirac semimetal candidate material.

\section{Results and Discussion}

$\mathrm{Ir}_{2} \mathrm{In}_{8} \mathrm{~S}$ crystallizes in a new structure type in the $P 4_{2} / \mathrm{mnm}$ space group (Figure 1). Open Quantum Materials Database (OQMD) calculations show that $\operatorname{Ir}_{2} \mathrm{In}_{8} \mathrm{~S}$ is a ternary phase that resides on the convex hull, with a formation enthalpy of $-0.266 \mathrm{eV} /$ atom (Figure $\mathrm{S} 6$ ). $\mathrm{Ir}_{2} \mathrm{In}_{8} \mathrm{~S}$ is a subsulfide, an unusual type of compound that lies between the classes of intermetallics and saltlike chalcogenides. Subsulfides contain metal-metal (Ir-In) along with metal-chalcogenide (In-S) interactions, causing the metal atoms to have very low formal oxidation states. Subchalcogenide materials are not a well-known class of compounds but they could be of great interest because of 
their potential for topological, superconducting and charge density wave (CDW) behavior, as their lower dimensional metallic substructures and exotic bonding motifs facilitate these interactions. ${ }^{31-}$ ${ }^{33}$ However, despite their promise for remarkable physical properties, it is a great challenge to synthesize and characterize new subchalcogenide materials, as they are notoriously difficult to form due to phase separation into constituent binary intermetallic and chalcogenide materials. Here, we overcome this difficulty by utilizing an indium metal flux to synthesize $\operatorname{Ir}_{2} \operatorname{In}_{8} S$, a method rarely used for these types of materials. ${ }^{34-35}$ Through this method, we can grow large single crystals on the scale of several millimeters that are stable in air, water, and dilute acids, making them ideal candidates for electrical transport measurements.

The structure of $\operatorname{Ir}_{2} \operatorname{In}_{8} \mathrm{~S}$ consists of a dense three-dimensional framework assembled by eight-coordinate $\operatorname{IrIn}_{8}$ polyhedra with chalcogenide atoms found in the channels along the $\mathrm{c}$ axis (crystallographic information in Tables S1-S3). The $\operatorname{IrIn}_{8}$ polyhedra are distorted bi-capped trigonal prisms that are corner sharing along the $a$ and $b$ axes but alternate between corner and edge sharing along the $c$ axis, resulting in the tetragonal nature of this compound. The sulfur atoms in the channels adopt an approximately square planar coordination with the surrounding indium atoms, forming long In-S bonds of approximately $2.8487(16) \AA$. The indium coordination environment is two Ir-In bonds (2.7397(14) $\AA$ ) along with a short In-In bond of approximately 2.689(3) A connecting the edge sharing polyhedra along the $c$ axis, and this bond length is shorter than the In-In dimer in $\operatorname{InS}(2.7624(4) \AA)$.

The chemical origin of subchalcogenide materials comes from an intermetallic network that has been partially oxidized by chalcogenide atoms, causing regions of metallic behavior and regions of ionic or salt-like behavior. In the case of $\operatorname{Ir}_{2} \operatorname{In}_{8} S$, the electronegative sulfide anions act as electron acceptors, taking electron density from the more electropositive indium atoms. In a 
sense, the indium is the bridging atom in the framework of the structure, as it interacts with both iridium and sulfur, but those two atoms do not directly bond with one another due to their large electronegativities. The fact that the chalcogenide ions in the channels act as ionic species is actually unusual for most subsulfides and subselenides, as they have shown in other structures that they can form polar covalent bonds with both chalcogenide atoms and transition metals. ${ }^{36-40}$ In a sense, the bonding in $\operatorname{Ir}_{2} \mathrm{In}_{8} \mathrm{~S}$ has more in common with subhalide materials, as the sulfur atoms act as counter ions that oxidize the intermetallic framework. ${ }^{41-43}$

To study the presence of topological behavior in $\operatorname{Ir}_{2} \operatorname{In}_{8} S$, we employed the CheckTopMat application from the Bilbao Crystallographic Server (BCS) and computed the irreducible representations (irreps) of the occupied bands at the high symmetry points $\Gamma$ and $Z$. This procedure checks whether the band structure is topological at the Fermi level. The calculations indicate that $\mathrm{Ir}_{2} \mathrm{In}_{8} \mathrm{~S}$ is an enforced semimetal (ES), which means that the semimetallic behavior is protected by symmetry. ${ }^{44}$ Two band crossings are observed along the line $\Lambda$ located only 0.025 and $0.040 \mathrm{eV}$ above the Fermi level, see Figure 2A-B. Following inversion or time reversal symmetry, there are two more crossings along the $\Gamma$-Z line. The electronic structure is similar to $\mathrm{Na}_{3} \mathrm{Bi}$ but with 4 total crossings instead of $2 .{ }^{4}$

We also computed the irreducible representations above the Fermi level along the high symmetry line $\Lambda$ connecting $\Gamma$ and $Z$ using Quantum Espresso to obtain information about the symmetry element which protects the crossing (see Figure 2C). ${ }^{45-47}$ Based on our group theoretical analysis, we identify this to be the $4_{2}$ screw axis, which differentiates the representations of the bands that cross and prevents their hybridization. Any perturbation that breaks this symmetry will enable hybridization of the bands and could open a gap. The general band dispersion of an anisotropic, tilted Dirac crossing is: 


$$
E(\vec{k})=\vec{w}_{0} \cdot \vec{k} \pm \sqrt{\left(v_{F}^{x} k_{x}\right)^{2}+\left(v_{F}^{y} k_{y}\right)^{2}+\left(v_{F}^{z} k_{z}\right)^{2}}
$$

Where $v_{F}^{i}$ is the $\mathrm{i}^{\text {th }}$ component of the Fermi velocity and $w_{0}$ is the tilt vector of the cone. ${ }^{48} \mathrm{We}$ performed ab-initio calculations and computed the band dispersion in the $y$ and $z$ directions centered at the crossings. A depiction of the lower tilted Dirac cone can be seen in Figure 2D, and fits and their values are displayed in Figure S5 and Table S4. Information about the Fermi velocity was extracted by fitting the bands to the dispersion given in Equation (1).

Inversion (and time reversal) symmetry dictates that band dispersion along $k_{x}>0$ must be the same as along the $k_{x}<0$ direction (the same holds for $k_{y}$ ). Since the crossings are located along the $\Gamma$-Z line $\left(k_{x}=k_{y}=0\right)$, the following must be true: $\omega_{0}{ }^{x}=\omega_{0}^{y}=0$. The 4-fold rotation axis along $z$ demands that $v_{F}^{x}=v_{F}^{y}$. Comparing the Fermi velocity values to the ones in $\mathrm{Na}_{3} \mathrm{Bi}$, we see that $v_{F}^{x, y}$ are 10 times smaller but $v_{F}^{z}$ is 10 times larger (Figure S5 and Table S4). The two Dirac points above the Fermi level in $\operatorname{Ir}_{2} \operatorname{In}_{8} S$ may contribute to some of the notable electronic properties found in $\operatorname{Ir}_{2} \operatorname{In}_{8} S$, as discussed below, such as the high carrier mobility and Shubnikov de-Haas (SdH) oscillations.

$\mathrm{Ir}_{2} \mathrm{In}_{8} \mathrm{~S}$ displays metallic resistivity (Figure 3A) and a negative Hall resistivity, suggesting that electrons are the majority carrier type (Figure $S 2)$. The residual resistivity ratio $\left(R R R, \rho_{300}\right.$ ${ }_{\mathrm{K}} / \rho_{1.8 \mathrm{~K}}$ ) for the crystals used for the Hall and quantum oscillations measurements seen below were 88 and 40, respectively, indicating high quality crystals with few intrinsic defects. $\operatorname{In} \operatorname{Ir}_{2} \operatorname{In}_{8} S$, the Hall resistivity as a function of magnetic field becomes noticeably nonlinear below $50 \mathrm{~K}$, suggesting an incorporation of hole carriers. To account for this, two-band fits of the data were taken into consideration when determining the Hall coefficients below $50 \mathrm{~K}$ (Table S5). The ntype carrier concentration of $\operatorname{Ir}_{2} \mathrm{In}_{8} \mathrm{~S}$ is approximately $10^{20} \mathrm{~cm}^{-3}$, and this value is relatively constant 
as a function of temperature. The electron and hole carrier mobilities of $\operatorname{Ir}_{2} \operatorname{In}_{8} S$ are high, with values of $\sim 10,000 \mathrm{~cm}^{2} \mathrm{~V}^{-1} \mathrm{~s}^{-1}$ at $1.8 \mathrm{~K}$ (Figure 3B). The low temperature carrier mobility of our sample of $\operatorname{Ir}_{2} \operatorname{In}_{8} S$ is comparable to that of the Weyl semimetal $\mathrm{WTe}_{2}\left(\sim 10^{4} \mathrm{~cm}^{2} \mathrm{~V}^{-1} \mathrm{~s}^{-1}\right)$, but significantly less than that of the monopnictides (e.g., TaP $\left.\mu \sim 10^{5} \mathrm{~cm}^{2} \mathrm{~V}^{-1} \mathrm{~s}^{-1}\right)$. ${ }^{49-51}$ The electron and hole carrier concentrations and mobilities are almost identical when fitted with the two-band model, suggesting that $\operatorname{Ir}_{2} \operatorname{In}_{8} S$ is a nearly fully compensated semimetal. ${ }^{50}$ The large carrier mobility values are likely a result of the invariance to backscattered electrons from the symmetry protected band crossings near the Fermi level, as the relaxation time $\tau$ is related to both mobility and drift velocity $v_{D}$

The magnetoresistance (MR) data in Figure 3C-D corroborate the high electron mobility of this structure, as the material has large, non-saturating $\mathrm{MR} \%$ of $6038 \%$ at $14 \mathrm{~T}$ and $3.34 \mathrm{~K}$ in a transverse configuration (I $\|[001]$, B $\|[110])$. Since magnetoresistance follows $\mathrm{MR}=1+(\mu B)^{2}$, where $\mu$ is mobility and $B$ is applied magnetic field), the fact that $\operatorname{Ir}_{2} \operatorname{In}_{8} S$ has a high $\mathrm{MR} \%$ is in line with its high carrier mobility. The MR\% differs greatly in sinusoidal fashion as the applied magnetic field direction changes, reaching a minimum of $225 \%$ when both current and field are along the $c$ axis in a longitudinal configuration (Figure 3D), suggesting that the overall Fermi surface is anisotropic. $\operatorname{Ir}_{2} \operatorname{In}_{8} \mathrm{~S}$ has a lower $\mathrm{MR} \%$ than the reported values of other fully compensated semimetals such as $\mathrm{WTe}_{2}, \mathrm{LaSb}$, and $\mathrm{TaAs}_{2} .{ }^{50,52-53}$ This modest MR\% may reflect a compromised crystal quality in comparison to crystals of the latter compounds with RRR in the $10^{2}-10^{3}$ range. We note, however, that a $3 \times 10^{5} \mathrm{MR} \%$ has been reported for compensated TaP with $\mathrm{RRR} \sim 65 .{ }^{49}$

Shubnikov de-Haas oscillations are shown in Figure 4. The full battery of angle and temperature dependent data revealed two small, predominant frequencies at $86\left(F_{\alpha 1}\right)$ and $155 \mathrm{~T}$ 
$\left(F_{\alpha 2}\right)$, as well as a third notable pocket at $241 \mathrm{~T}\left(F_{\beta}\right)$. The strongest frequency, $F_{\alpha}$, has one harmonic $\left(F_{2 \alpha 1}\right)$ at $171 \mathrm{~T}$ and $F_{\alpha 2}$ has one harmonic $\left(F_{2 \alpha 2}\right)$ at $303 \mathrm{~T} . \alpha_{1}$ and $\alpha_{2}$ do not vary strongly as a function of angle, suggesting that these pockets are isotropic in nature despite the calculated Fermi surface predicting a more ellipsoidal topology for both (Figures S3 and S4). For $F_{\alpha l}$, we can use the Lifshitz-Onsager relation to determine the extremal cross-sectional area $\left(S_{F}\right)$ of the Fermi surface, Equation (2)

$$
F=\frac{\hbar}{2 \pi e} S_{F}
$$

where $F$ is the frequency of the pocket and $h$ is Planck's constant. ${ }^{54} \operatorname{Ir}_{2} \operatorname{In}_{8} \mathrm{~S}$ has an $S_{F}$ of 0.00821 $\AA^{-2}$, which occupies $2.04 \%$ of the Brillouin zone area along the $k_{x}-k_{y}$ plane, and a Fermi momentum $k_{F}=\sqrt{\frac{S_{F}}{\pi}}=0.051 \AA^{-1} . F_{\alpha l}$ and $F_{\alpha 2}$ survive up to $20 \mathrm{~K}$, while $F_{\beta}$ is still visible at $15 \mathrm{~K}$. We can fit the decay amplitude of each frequency to determine the effective mass of the pocket by the Lifshitz-Kosevich (LK) formula:

$$
A(T, B) \sim e^{\frac{-2 \pi^{2} k_{B} T_{D}}{\hbar \omega_{C}}} \frac{\frac{2 \pi^{2} k_{B} T}{\hbar \omega_{C}}}{\sinh \frac{2 \pi^{2} k_{B} T}{\hbar \omega_{C}}}
$$

where $k_{B}$ is Boltzmann's constant, $T_{D}$ is the Dingle temperature, $B$ is applied field, $T$ is temperature, and $\omega_{C}$ is the cyclotron frequency $\left(\omega_{C}=e B / m^{*}\right.$, with $m^{*}$ as the carrier effective mass). The value of $B$ for fitting was the average of the minimum and maximum value in the Fast Fourier Transform data. The LK fit shown in Figure 4C reveals an $\mathrm{m}^{*}$ of $0.234(6) m_{e}$ for $F_{\alpha l}$, with $m_{e}$ being the mass of a free electron in vacuum. $F_{\alpha 2}$ has a nearly identical fitted effective mass to $F_{\alpha 1}\left(m^{*}=\right.$ $\left.0.234(4) m_{e}\right)$, and $F_{\beta}, F_{\delta}$, and $F_{\gamma}$ have notably heavier fitted effective masses $\left(0.367(6) m_{e}\right.$, $0.42(2) m_{e}$, and $0.34(1) m_{e}$, respectively). The low effective masses of the two primary alpha frequencies are coincident with the high electron carrier mobility and persistence of $\mathrm{SdH}$ 
oscillations up to $20 \mathrm{~K}$. The prominent $\alpha_{1}$ and $\alpha_{2}$ pockets have cross sectional areas of 0.00821 and $0.0148 \mathrm{~A}^{-2}$, respectively, which are consistent with the calculated cross sectional areas of 0.00815 and $0.0120 \AA^{-2}$ from the Fermi surface calculations (see Figure S4).

To explore the possibility of topological behavior, the Landau level (LL) fan diagram can be plotted to approximate the value of the Berry phase by indexing the peaks and valleys of the $F_{\alpha l}$ frequency after applying a band pass filter to the data. The Onsager phase $(\gamma)$ is determined by the equation $\gamma=1 / 2-\varphi_{B}$, where $\varphi_{B}$ is the Berry phase. ${ }^{54}$ The intercept of the LL fan diagram can be used to determine $\gamma$, which can then extract the value of $\varphi_{B}$. For $\operatorname{Ir}_{2} \mathrm{In}_{8} \mathrm{~S}$, we obtain an intercept of 0.03 , which might suggest a topologically nontrivial Berry phase value of $\sim \pi$. We note that the Landau fan diagram derived from our data only reaches a minimum Landau level of 6, which is far from the quantum limit and would require much higher applied magnetic fields to definitively verify this claim.

\section{Conclusion}

In conclusion, $\mathrm{Ir}_{2} \mathrm{In}_{8} \mathrm{~S}$ is a novel Dirac semimetal candidate that crystallizes in a new structure type. $\operatorname{Ir}_{2} \mathrm{In}_{8} \mathrm{~S}$ is a subsulfide: A rare, metal-rich compound that contains both metal-metal and metal-chalcogenide bonding interactions. The symmetry analysis of its electronic band structure indicates that it has two symmetry-protected crossings next to each other along the $\Gamma-Z$ direction. Analysis of the $\mathrm{SdH}$ oscillations show several small Fermi surface pockets, a small effective mass, and the possibility of a nontrivial Berry phase in the electronic structure. These experimental data, along with the theoretical analysis of its band structure, point to the topological character of this material. $\operatorname{Ir}_{2} \operatorname{In}_{8} S$ is therefore a notable new entry in the field of topological semimetals, as it is not found in any database and offers a new system for future studies on its quantum properties. 


\section{Experimental Section}

Synthesis. For $\operatorname{Ir}_{2} \operatorname{In}_{8} \mathrm{~S}$, Ir (1 mmol, $\left.0.1922 \mathrm{~g}\right)$, S (0.5 mmol, $\left.0.0163 \mathrm{~g}\right)$, and In (20 mmol, $2.2964 \mathrm{~g})$ were loaded into alumina crucibles. A 100-mesh stainless steel filter was placed on top of the alumina crucibles, and a small piece of alumina tubing was placed on top of the filter as a counterweight. The crucible, filter, and tubing were loaded into $18 \mathrm{~mm}$ fused silica tubes and sealed under vacuum at $\sim 3 \times 10^{-3}$ mbar, heated to $1000^{\circ} \mathrm{C}$ in $12 \mathrm{~h}$, held there for $24 \mathrm{~h}$, and cooled to $650{ }^{\circ} \mathrm{C}$ in $24-48 \mathrm{~h}$. After being held at $650^{\circ} \mathrm{C}$ for a few hours, the tubes were removed from the furnace and immediately centrifuged to remove excess indium flux. Residual indium on the surface of the crystals was removed by etching in dilute (10\% by weight) $\mathrm{HCl}$ for $2-3$ hours, and then the crystals were filtered and washed with water and acetone. The crystals are stable in air and stable in dilute $\mathrm{HCl}$ for at least 2-3 days. Yields for these reactions range from $50-75 \%$ by weight depending on the limiting reagent.

Single Crystal X-ray Diffraction. Crystals of $\operatorname{Ir}_{2} \mathrm{In}_{8} \mathrm{~S}$ were adhered to a glass fiber with super glue. Single crystal x-ray diffraction was performed at $200 \mathrm{~K}$ on a Bruker-APEX II CCD diffractometer with MoK $\alpha$ radiation $(\lambda=0.71073 \AA)$. The data were integrated using SAINTv8.38A and the multi-scan absorption correction was applied using SADABS. ${ }^{55}$ The structures were solved with intrinsic phasing in the SHELXT software package, and refined with SHELXL by the Least Squares method. ${ }^{56}$ The crystallographic information for the structures can be found in Tables S1-S3. Additional crystallographic information can be found in the supporting information.

Density Functional Theory (DFT) Calculations. Band structure calculations were performed using Density Functional Theory (DFT) as implemented in the Vienna Ab initio Simulation Package (VASP). ${ }^{57-60}$ The interaction between ion cores and valence electrons was 
treated by the projector augmented-wave method (PAW), the generalized gradient approximation (GGA) for the exchange-correlation potential with the Perdew-Burke-Ernkzerhof for solids parametrization and spin- orbit coupling (SOC) was taken into account by the second variation method. ${ }^{61-63}$ A Monkhorst-Pack k-point grid of $(6 \times 6 \times 6)$ for reciprocal space integration and 500 $\mathrm{eV}$ energy cutoff of the plane-wave expansion have been used. Atomic positions were fixed and therefore the symmetries of the space groups were preserved without disorder. The Open Quantum Materials Database (OQMD) was used to perform stability calculations on $\operatorname{Ir}_{2} \operatorname{In}_{8} S$.

Charge Transport. Temperature-variable resistivity and Hall effect measurements on single crystals (approximate dimensions: $1 \times 1 \times 0.3 \mathrm{~mm}^{3}$ ) were conducted on a Quantum Design Dynacool Physical Property Measurement System (PPMS) between 1.8 and 300 K. Resistivity was measured in a 4-point collinear geometry (with current in an arbitrary direction along the ab plane) and the Hall effect measured using two Hall voltage contacts placed perpendicular to the axis of current flow. The magnetic field was applied perpendicular to the axis of current flow from -9 to +9 T. Temperature and field were cycled multiple times to confirm data reproducibility. Electronic resistivity and Hall effect measurements were performed simultaneously on the same sample. Stable, Ohmic contact was achieved by thermally evaporating Au metal pads, or sputtering Pt metal pads on the sample and attaching Au wires with silver paste (Dupont 4929N). The residual resistivity ratio (RRR, $\rho_{300 \mathrm{~K}} / \rho_{1.8 \mathrm{~K}}$ ) for this crystal was $\sim 88$. Multiband fits for the low temperature data used a simple two-band model that has been described elsewhere. ${ }^{64}$

To perform SdH Oscillations, single crystals were oriented such that current was along the c axis and the perpendicular axis was the (110) plane. Samples were measured on a Quantum Design PPMS with a $14 \mathrm{~T}$ magnet attachment between $3.34 \mathrm{~K}$ and $25 \mathrm{~K}$. Resistivity was again measured in a 4-point collinear geometry. The magnetic field was initially applied perpendicular 
to the axis of current flow from 5 to $14 \mathrm{~T}$ to measure magnetoresistance as a function of field, revealing the SdH oscillations. On the PPMS rotator, the MR was measured from 0 to 100 degrees in 10 degree increments through the same range. The RRR $\left(\rho_{300 \mathrm{~K}} / \rho_{3.34 \mathrm{~K}}\right)$ for this oriented crystal was $\sim 40$. A band pass filter was applied using OriginLab 8 to isolate the $\mathrm{SdH}$ frequency used to create the Landau fan diagram.

\section{Associated Content}

The supporting information is available free of charge via the internet at the ACS Publications website.

Experimental details, SEM/EDS, Hall resistivity and fitting information, FFT data, additional Fermi surface and band calculations, and additional crystallographic information (CIF)

\section{Author Information \\ Corresponding Author \\ *E-mail: $\underline{\text { m-kanatzidis@northwestern.edu }}$}

Note: The authors declare no competing financial interests.

\section{Acknowledgements}

This work was supported by the National Science Foundation (NSF) grant DMR-1708254 (synthesis and structural characterization). Transport and magnetic property measurements were performed at Argonne National Laboratory supported by the U.S. Department of Energy (DOE), Office of Science, Basic Energy Sciences (BES), Materials Sciences and Engineering Division. Single crystal diffraction data was performed at the IMSERC facility at Northwestern University, which has received support from the Soft and Hybrid Nanotechnology Experimental (SHyNE) Resource (NSF NNCI-1542205); the State of Illinois; and International Institute for 
Nanotechnology (IIN). The work for preliminary DFT calculations and partial density of states calculations carried out by J.E.P. and C.W. was supported by the U.S. Department of Energy, Office of Science Basic Energy Sciences grant DE-SC0014520. L.M.S. was supported by NSF through the Princeton Center for Complex Materials, a Materials Research Science and Engineering Center DMR-1420541, and by a MURI grant on Topological Insulators from the Army Research Office, grant number ARO W911NF-12-1-0461. M.G.V. and A.B. acknowledge the IS2016-75862-P and FIS2016-76617-P national projects of the Spanish MINECO and the Department of Education, Universities and Research of the Basque Government and the University of the Basque Country (IT756-13). We acknowledge QUEST, a supercomputer facility at Northwestern University. We thank Dr. Ido Hadar and Daniel G. Chica for assisting in gold evaporation on the samples prior to transport measurements. We also thank Dr. Constantinos C. Stoumpos and Dr. Kyle M. McCall for helpful discussions.

\section{References:}

1. $\quad$ Liu, Z. K.; Jiang, J.; Zhou, B.; Wang, Z. J.; Zhang, Y.; Weng, H. M.; Prabhakaran, D.; Mo, S. K.; Peng, H.; Dudin, P.; Kim, T.; Hoesch, M.; Fang, Z.; Dai, X.; Shen, Z. X.; Feng, D. L.; Hussain, Z.; Chen, Y. L. A stable three-dimensional topological Dirac semimetal $\mathrm{Cd}_{3} \mathrm{As}_{2}$. Nat. Mater. 2014, 13, 677.

2. Moll, P. J. W.; Nair, N. L.; Helm, T.; Potter, A. C.; Kimchi, I.; Vishwanath, A.; Analytis, J. G. Transport evidence for Fermi-arc-mediated chirality transfer in the Dirac semimetal $\mathrm{Cd}_{3} \mathrm{As}_{2}$. Nature 2016, 535, 266.

3. Liang, T.; Gibson, Q.; Ali, M. N.; Liu, M.; Cava, R. J.; Ong, N. P. Ultrahigh mobility and giant magnetoresistance in the Dirac semimetal $\mathrm{Cd}_{3} \mathrm{As}_{2}$. Nat. Mater. 2014, 14, 280.

4. $\quad$ Liu, Z. K.; Zhou, B.; Zhang, Y.; Wang, Z. J.; Weng, H. M.; Prabhakaran, D.; Mo, S. K.; Shen, Z. X.; Fang, Z.; Dai, X.; Hussain, Z.; Chen, Y. L. Discovery of a Three-Dimensional Topological Dirac Semimetal, $\mathrm{Na}_{3} \mathrm{Bi}$. Science 2014, 343, 864. 
5. Borisenko, S.; Gibson, Q.; Evtushinsky, D.; Zabolotnyy, V.; Büchner, B.; Cava, R. J. Experimental Realization of a Three-Dimensional Dirac Semimetal. Phys. Rev. Lett. 2014, 113, 027603.

6. Schoop, L. M.; Pielnhofer, F.; Lotsch, B. V. Chemical Principles of Topological Semimetals. Chem. Mater. 2018, 30, 3155-3176.

7. Weng, H.; Dai, X.; Fang, Z. Topological semimetals predicted from first-principles calculations. J. Phys. Condens. Mat. 2016, 28, 303001.

8. Schoop, L. M.; Topp, A.; Lippmann, J.; Orlandi, F.; Müchler, L.; Vergniory, M. G.; Sun, Y.; Rost, A. W.; Duppel, V.; Krivenkov, M.; Sheoran, S.; Manuel, P.; Varykhalov, A.; Yan, B.; Kremer, R. K.; Ast, C. R.; Lotsch, B. V. Tunable Weyl and Dirac states in the nonsymmorphic compound CeSbTe. Sci. Adv. 2018, 4, eaar2317.

9. Shekhar, C.; Nayak, A. K.; Sun, Y.; Schmidt, M.; Nicklas, M.; Leermakers, I.; Zeitler, U.; Skourski, Y.; Wosnitza, J.; Liu, Z.; Chen, Y.; Schnelle, W.; Borrmann, H.; Grin, Y.; Felser, C.; Yan, B. Extremely large magnetoresistance and ultrahigh mobility in the topological Weyl semimetal candidate NbP. Nat. Phys. 2015, 11, 645.

10. Schnelle, W.; Leithe-Jasper, A.; Rosner, H.; Schappacher, F. M.; Pöttgen, R.; Pielnhofer, F.; Weihrich, R. Ferromagnetic ordering and half-metallic state of $\mathrm{Sn}_{2} \mathrm{Co}_{3} \mathrm{~S}_{2}$ with the shanditetype structure. Phys. Rev. B 2013, 88, 144404.

11. Liu, E.; Sun, Y.; Kumar, N.; Muechler, L.; Sun, A.; Jiao, L.; Yang, S.-Y.; Liu, D.; Liang, A.; Xu, Q.; Kroder, J.; Süß, V.; Borrmann, H.; Shekhar, C.; Wang, Z.; Xi, C.; Wang, W.; Schnelle, W.; Wirth, S.; Chen, Y.; Goennenwein, S. T. B.; Felser, C. Giant anomalous Hall effect in a ferromagnetic kagome-lattice semimetal. Nat. Phys. 2018, 14, 1125-1131.

12. Wang, Q.; Xu, Y.; Lou, R.; Liu, Z.; Li, M.; Huang, Y.; Shen, D.; Weng, H.; Wang, S.; Lei, H. Large intrinsic anomalous Hall effect in half-metallic ferromagnet $\mathrm{Co}_{3} \mathrm{Sn}_{2} \mathrm{~S}_{2}$ with magnetic Weyl fermions. Nat. Comm. 2018, 9, 3681.

13. Huang, X.; Zhao, L.; Long, Y.; Wang, P.; Chen, D.; Yang, Z.; Liang, H.; Xue, M.; Weng, H.; Fang, Z.; Dai, X.; Chen, G. Observation of the Chiral-Anomaly-Induced Negative Magnetoresistance in 3D Weyl Semimetal TaAs. Phys. Rev. X 2015, 5, 031023.

14. Weber, C. P.; Schoop, L. M.; Parkin, S. S. P.; Newby, R. C.; Nateprov, A.; Lotsch, B.; Mariserla, B. M. K.; Kim, J. M.; Dani, K. M.; Bechtel, H. A.; Arushanov, E.; Ali, M. Directly photoexcited Dirac and Weyl fermions in ZrSiS and NbAs. Appl. Phys. Lett. 2018, 113, 221906. 15. Horio, M.; Matt, C. E.; Kramer, K.; Sutter, D.; Cook, A. M.; Sassa, Y.; Hauser, K.; Månsson, M.; Plumb, N. C.; Shi, M.; Lipscombe, O. J.; Hayden, S. M.; Neupert, T.; Chang, J. Two-dimensional type-II Dirac fermions in layered oxides. Nat. Comm. 2018, 9, 3252.

16. Fei, F.; Bo, X.; Wang, P.; Ying, J.; Li, J.; Chen, K.; Dai, Q.; Chen, B.; Sun, Z.; Zhang, M.; Qu, F.; Zhang, Y.; Wang, Q.; Wang, X.; Cao, L.; Bu, H.; Song, F.; Wan, X.; Wang, B. Band Structure Perfection and Superconductivity in Type-II Dirac Semimetal $\operatorname{Ir}_{1-\mathrm{x}} \mathrm{Pt}_{\mathrm{x}} \mathrm{Te}_{2}$. Adv. Mater. 2018, 30, 1801556.

17. Kobayashi, S.; Sato, M. Topological Superconductivity in Dirac Semimetals. Phys. Rev. Lett. 2015, 115, 187001.

18. Yan, M.; Huang, H.; Zhang, K.; Wang, E.; Yao, W.; Deng, K.; Wan, G.; Zhang, H.; Arita, M.; Yang, H.; Sun, Z.; Yao, H.; Wu, Y.; Fan, S.; Duan, W.; Zhou, S. Lorentz-violating type-II Dirac fermions in transition metal dichalcogenide PtTe 2 . Nat. Comm. 2017, 8, 257. 19. Khan, M. A.; Graf, D. E.; Vekhter, I.; Browne, D. A.; DiTusa, J. F.; Phelan, W. A.; Young, D. P. Quantum oscillations and a nontrivial Berry phase in the noncentrosymmetric topological superconductor candidate BiPd. Phys. Rev. B 2019, 99, 020507. 
20. Fei, F.; Bo, X.; Wang, R.; Wu, B.; Jiang, J.; Fu, D.; Gao, M.; Zheng, H.; Chen, Y.; Wang, X.; Bu, H.; Song, F.; Wan, X.; Wang, B.; Wang, G. Nontrivial Berry phase and type-II Dirac transport in the layered material PdTe 2 . Phys. Rev. B 2017, 96, 041201.

21. Noh, H.-J.; Jeong, J.; Cho, E.-J.; Kim, K.; Min, B. I.; Park, B.-G. Experimental Realization of Type-II Dirac Fermions in a PdTe 2 Superconductor. Phys. Rev. Lett. 2017, 119, 016401.

22. Zunger, A. Beware of plausible predictions of fantasy materials. Nature 2019, 566, 447449.

23. Klemenz, S.; Lei, S.; Schoop, L. M. Topological Semimetals in Square-Net Materials. Annu. Rev. Mater. Res. 2019.

24. Zhu, Z.; Winkler, G. W.; Wu, Q.; Li, J.; Soluyanov, A. A. Triple Point Topological Metals. Phys. Rev. X 2016, 6, 031003.

25. Weng, H.; Fang, C.; Fang, Z.; Dai, X. Topological semimetals with triply degenerate nodal points in $\theta$-phase tantalum nitride. Phys. Rev. B 2016, 93, 241202.

26. Tang, F.; Po, H. C.; Vishwanath, A.; Wan, X. Comprehensive search for topological materials using symmetry indicators. Nature 2019, 566, 486-489.

27. Zhang, T.; Jiang, Y.; Song, Z.; Huang, H.; He, Y.; Fang, Z.; Weng, H.; Fang, C. Catalogue of topological electronic materials. Nature 2019, 566, 475-479.

28. Vergniory, M. G.; Elcoro, L.; Felser, C.; Regnault, N.; Bernevig, B. A.; Wang, Z. A complete catalogue of high-quality topological materials. Nature 2019, 566, 480-485.

29. Gui, X.; Pletikosic, I.; Cao, H.; Tien, H.-J.; Xu, X.; Zhong, R.; Wang, G.; Chang, T.-R.; Jia, S.; Valla, T.; Xie, W.; Cava, R. J. A New Magnetic Topological Quantum Material Candidate by Design. ACS Cent. Sci. 2019, 5, 900-910.

30. Savage, N. Topology Shapes a Search for New Materials. ACS Cent. Sci. 2018, 4, 523526.

31. Rasche, B.; Isaeva, A.; Ruck, M.; Borisenko, S.; Zabolotnyy, V.; Büchner, B.; Koepernik, K.; Ortix, C.; Richter, M.; van den Brink, J. Stacked topological insulator built from bismuth-based graphene sheet analogues. Nat. Mater. 2013, 12, 422.

32. Sakamoto, T.; Wakeshima, M.; Hinatsu, Y.; Matsuhira, K. Charge-density-wave superconductor $\mathrm{Bi}_{2} \mathrm{Rh}_{3} \mathrm{Se}_{2}$. Phys. Rev. B 2007, 75, 060503.

33. Sakamoto, T.; Wakeshima, M.; Hinatsu, Y.; Matsuhira, K. Transport properties in normal-metal $\mathrm{Bi}_{2} \mathrm{Pd}_{3} \mathrm{~S}_{2}$ and superconducting $\mathrm{Bi}_{2} \mathrm{Pd}_{3} \mathrm{Se}_{2}$. Phys. Rev. B 2008, 78, 024509.

34. Canfield, P. C.; Kong, T.; Kaluarachchi, U. S.; Jo, N. H. Use of frit-disc crucibles for routine and exploratory solution growth of single crystalline samples. Philos. Mag. 2016, 96, 8492.

35. Lin, X.; Bud'ko, S. L.; Canfield, P. C. Development of viable solutions for the synthesis of sulfur bearing single crystals. Philos. Mag. 2012, 92, 2436-2447.

36. Baranov, A. I.; Isaeva, A. A.; Kloo, L.; Popovkin, B. A. New Metal-Rich Sulfides $\mathrm{Ni}_{6} \mathrm{SnS}_{2}$ and $\mathrm{Ni}_{9} \mathrm{Sn}_{2} \mathrm{~S}_{2}$ with a $2 \mathrm{D}$ Metal Framework: Synthesis, Crystal Structure, and Bonding. Inorg. Chem. 2003, 42, 6667-6672.

37. Baranov, A. I.; Kloo, L.; Olenev, A. V.; Popovkin, B. A.; Romanenko, A. I.; Shevelkov, A. V. Unique Metallic Wires in a Novel Quasi-1D Compound. Synthesis, Crystal and Electronic Structure, and Properties of $\mathrm{Ni}_{8} \mathrm{Bi}_{8}$ SI. J. Am. Chem. Soc. 2001, 123, 12375-12379.

38. Chen, H. Y.; Tuenge, R. T.; Franzen, H. F. Preparation and crystal structure of $\mathrm{Nb}_{14} \mathrm{~S}_{5}$. Inorg. Chem. 1973, 12, 552-555. 
39. Cheng, H. Y.; Franzen, H. F. The crystal structure of $\mathrm{Zr}_{9} \mathrm{~S}_{2}$. Acta Cryst. B 1972, 28, 13991404.

40. Franzen, H. F. Structure and bonding in metal-rich compounds: Pnictides, chalcides and halides. Prog. Solid State Chem. 1978, 12, 1-39.

41. Ruck, M. $\mathrm{Bi}_{5.6} \mathrm{Ni}_{5} \mathrm{I}$ : Eine partiell oxidierte intermetallische Phase mit Kanalstruktur. $Z$. Anorg. Allg. Chem. 1995, 621, 2034-2042.

42. Ruck, M. From the Metal to the Molecule_-Ternary Bismuth Subhalides. Angew. Chem., Int. Ed. 2001, 40, 1182-1193.

43. Ruck, M. At the edge of covalency: metallic nanotubes, low-dimensional metals and clusters. Z. Kristallogr. Cryst. Mater. 2010, 225, 167.

44. Bilbao Crystallographic Server, 'Check topological mat', http://www.cryst.ehu.es/cgibin/cryst/programs/topological.pl. 2018.

45. van Setten, M. J.; Giantomassi, M.; Bousquet, E.; Verstraete, M. J.; Hamann, D. R.; Gonze, X.; Rignanese, G. M. The PseudoDojo: Training and grading a 85 element optimized norm-conserving pseudopotential table. Comput. Phys. Commun. 2018, 226, 39-54.

46. Giannozzi, P.; Andreussi, O.; Brumme, T.; Bunau, O.; Buongiorno Nardelli, M.; Calandra, M.; Car, R.; Cavazzoni, C.; Ceresoli, D.; Cococcioni, M.; Colonna, N.; Carnimeo, I.; Dal Corso, A.; de Gironcoli, S.; Delugas, P.; DiStasio, R. A.; Ferretti, A.; Floris, A.; Fratesi, G.; Fugallo, G.; Gebauer, R.; Gerstmann, U.; Giustino, F.; Gorni, T.; Jia, J.; Kawamura, M.; Ko, H. Y.; Kokalj, A.; Küçükbenli, E.; Lazzeri, M.; Marsili, M.; Marzari, N.; Mauri, F.; Nguyen, N. L.; Nguyen, H. V.; Otero-de-la-Roza, A.; Paulatto, L.; Poncé, S.; Rocca, D.; Sabatini, R.; Santra, B.; Schlipf, M.; Seitsonen, A. P.; Smogunov, A.; Timrov, I.; Thonhauser, T.; Umari, P.; Vast, N.; Wu, X.; Baroni, S. Advanced capabilities for materials modelling with Quantum ESPRESSO. J. Phys. Condens. Mat. 2017, 29, 465901.

47. Giannozzi, P.; Baroni, S.; Bonini, N.; Calandra, M.; Car, R.; Cavazzoni, C.; Ceresoli, D.; Chiarotti, G. L.; Cococcioni, M.; Dabo, I.; Dal Corso, A.; de Gironcoli, S.; Fabris, S.; Fratesi, G.; Gebauer, R.; Gerstmann, U.; Gougoussis, C.; Kokalj, A.; Lazzeri, M.; Martin-Samos, L.; Marzari, N.; Mauri, F.; Mazzarello, R.; Paolini, S.; Pasquarello, A.; Paulatto, L.; Sbraccia, C.; Scandolo, S.; Sclauzero, G.; Seitsonen, A. P.; Smogunov, A.; Umari, P.; Wentzcovitch, R. M. QUANTUM ESPRESSO: a modular and open-source software project for quantum simulations of materials. J. Phys. Condens. Mat. 2009, 21, 395502.

48. Goerbig, M. O.; Fuchs, J. N.; Montambaux, G.; Piéchon, F. Tilted anisotropic Dirac cones in quinoid-type graphene and $\alpha-(\text { BEDT-TTF })_{2} \mathrm{I}_{3}$. Phys. Rev. B 2008, 78, 045415.

49. Hu, J.; Liu, J. Y.; Graf, D.; Radmanesh, S. M. A.; Adams, D. J.; Chuang, A.; Wang, Y.; Chiorescu, I.; Wei, J.; Spinu, L.; Mao, Z. Q. $\pi$ Berry phase and Zeeman splitting of Weyl semimetal TaP. Sci. Rep. 2016, 6, 18674.

50. $\quad$ Ali, M. N.; Schoop, L. M.; Xiong, J.; Flynn, S.; Gibson, Q.; Hirschberger, M.; Ong, N. P.; Cava, R. J. Correlation of crystal quality and extreme magnetoresistance of $\mathrm{WTe}_{2}$. Europhys. Lett. 2015, 110, 67002.

51. Ali, M. N.; Xiong, J.; Flynn, S.; Tao, J.; Gibson, Q. D.; Schoop, L. M.; Liang, T.; Haldolaarachchige, N.; Hirschberger, M.; Ong, N. P.; Cava, R. J. Large, non-saturating magnetoresistance in $\mathrm{WTe}_{2}$. Nature 2014, 514, 205.

52. Zeng, L. K.; Lou, R.; Wu, D. S.; Xu, Q. N.; Guo, P. J.; Kong, L. Y.; Zhong, Y. G.; Ma, J. Z.; Fu, B. B.; Richard, P.; Wang, P.; Liu, G. T.; Lu, L.; Huang, Y. B.; Fang, C.; Sun, S. S.; Wang, Q.; Wang, L.; Shi, Y. G.; Weng, H. M.; Lei, H. C.; Liu, K.; Wang, S. C.; Qian, T.; Luo, J. 
L.; Ding, H. Compensated Semimetal LaSb with Unsaturated Magnetoresistance. Phys. Rev. Lett. 2016, 117, 127204.

53. Yuan, Z.; Lu, H.; Liu, Y.; Wang, J.; Jia, S. Large magnetoresistance in compensated semimetals $\mathrm{TaAs}_{2}$ and $\mathrm{NbAs}_{2}$. Phys. Rev. B 2016, 93, 184405.

54. Shoenberg, D., Magnetic oscillations in metals. Cambridge University Press: London, 1984.

55. SAINT, version 8.38A; Bruker Analytical X-ray Instruments, Inc.: Madison, WI. SAINT, version 8.38A; Bruker Analytical X-ray Instruments, Inc.: Madison, WI. 2008.

56. Sheldrick, G. M. SHELXTL, version 6.14; Bruker Analytical X-ray Instruments, Inc.: Madison, WI. SHELXTL, version 6.14; Bruker Analytical X-ray Instruments, Inc.: Madison, WI. 2003.

57. Kohn, W. S., L.J. Self-Consistent Equations Including Exchange and Correlation Effects. Phys. Rev. 1965, 140, A1133.

58. Hohenberg, P.; Kohn, W. Inhomogeneous Electron Gas. Phys. Rev. 1964, 136, B864B871.

59. Kresse, G.; Furthmüller, J. Efficient iterative schemes for ab initio total-energy calculations using a plane-wave basis set. Phys. Rev. B 1996, 54, 11169-11186.

60. Kresse, G.; Hafner, J. Ab initio molecular dynamics for open-shell transition metals. Phys. Rev. B 1993, 48, 13115-13118.

61. Kresse, G.; Joubert, D. From ultrasoft pseudopotentials to the projector augmented-wave method. Phys. Rev. B 1999, 59, 1758-1775.

62. Perdew, J. P.; Burke, K.; Ernzerhof, M. Generalized Gradient Approximation Made Simple. Phys. Rev. Lett. 1996, 77, 3865-3868.

63. Hobbs, D.; Kresse, G.; Hafner, J. Fully unconstrained noncollinear magnetism within the projector augmented-wave method. Phys. Rev. B 2000, 62, 11556-11570.

64. Ghimire, N. J.; Khan, M. A.; Botana, A. S.; Jiang, J. S.; Mitchell, J. F. Anisotropic angular magnetoresistance and Fermi surface topology of the candidate novel topological metal $\mathrm{Pd}_{3}$ Pb. Phys. Rev. Mater. 2018, 2, 081201. 
A
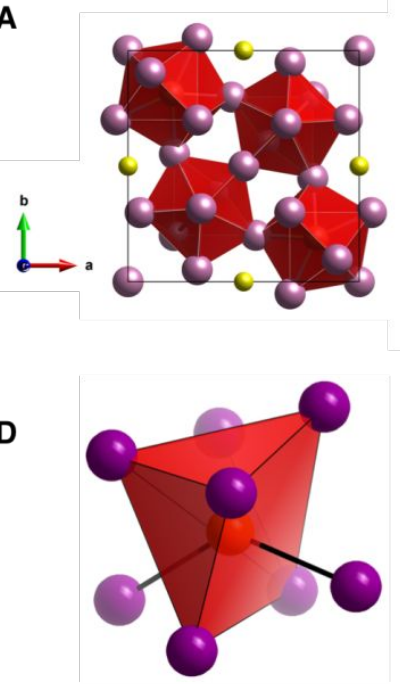
polyhedra along the $c$ axis.
B
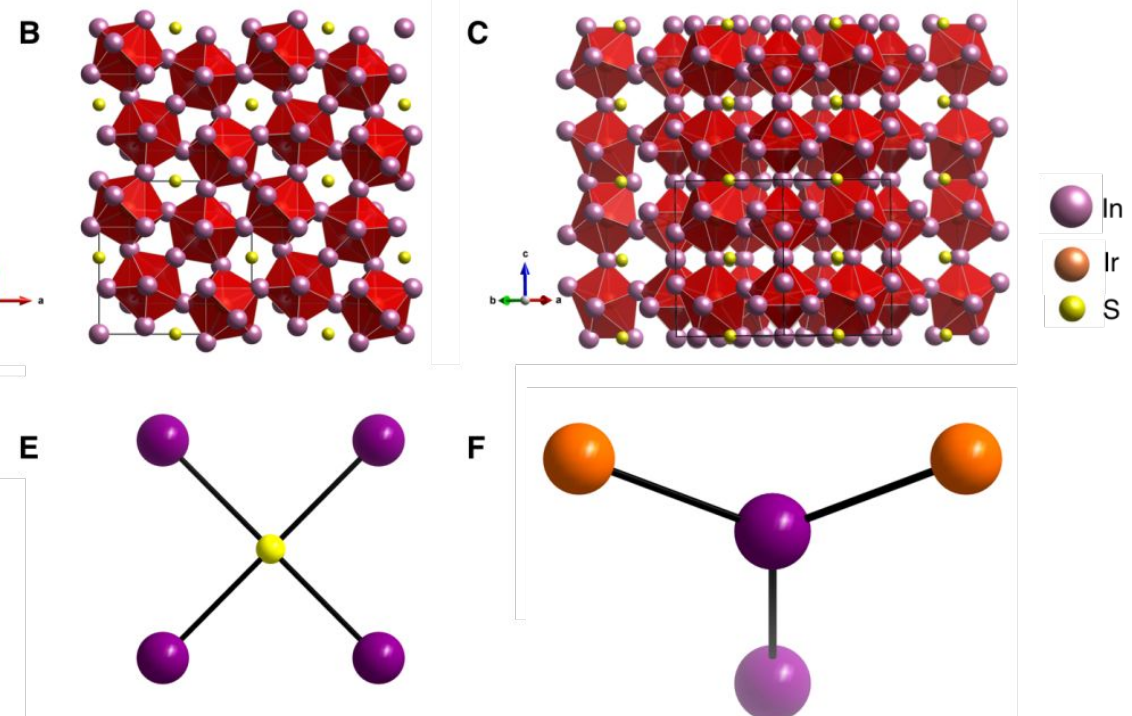

$\mathbf{F}$

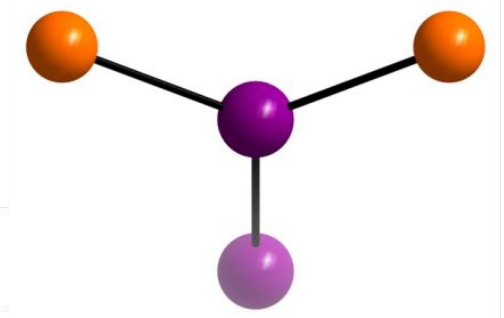

Figure 1: The structure of $\operatorname{Ir}_{2} \mathrm{In}_{8} \mathrm{~S}$. (A) Individual unit cell of $\operatorname{Ir}_{2} \mathrm{In}_{8} \mathrm{~S}$ along the $c$ axis. The space group is $P 4_{2} / \mathrm{mnm}$, and the lattice parameters are $a=9.8937(16) \AA, c=10.0991(17) \AA, \alpha=\beta=\gamma=90^{\circ}$. (B, C) Extended structure along the $a b$ plane (B) and the $c$ axis (C). The connectivity along the $c$ axis alternates between corner and edge sharing polyhedra. (D) Individual $\operatorname{IrIn}_{8}$ coordination environment, showcasing the distorted bi-capped trigonal prism geometry. (E) Coordination environment of sulfur, adopting an approximately square planar geometry with In-S bonds $(2.8487(16) \AA$ ). (F) Coordination environment of indium, showcasing the Ir-In bonds (2.7397(14) $\AA$ ) and In-In bond (2.689(3) $\AA)$ that forms the edge sharing 
2

3

4

5

6

7

8
A
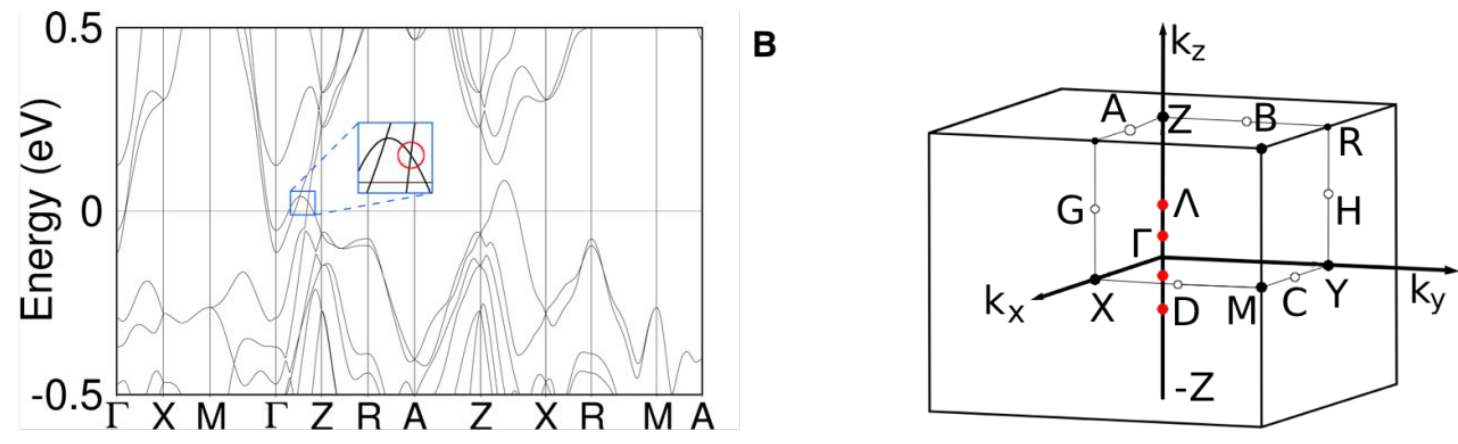

C

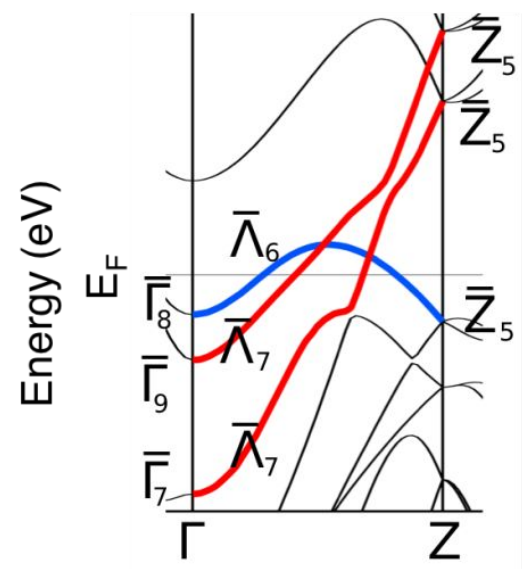

B

D

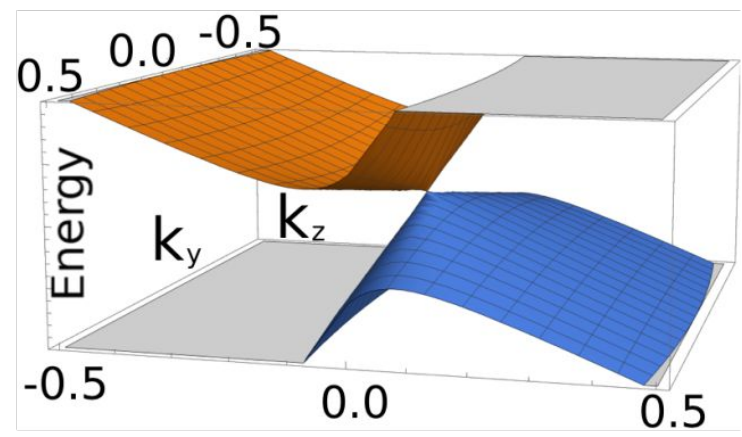

Figure 2: (A) $\operatorname{Ir}_{2} \mathrm{In}_{8} \mathrm{~S}$ band structure. All band structures have two Dirac crossings in the line $\Gamma-Z$. Highest fitted values correspond to the crossing inside the red circle in $\operatorname{Ir}_{2} \mathrm{In}_{8} \mathrm{~S}$ band structure. (B) Brillouin zone of the space group $P 4_{2} / m n m$ (136). The two Dirac crossings near the Fermi level are depicted as red dots. (C) Irreducible representations (irreps) at high-symmetry points and the line connecting them. Blue color stands for $\Lambda_{6}$ irrep and red color for $\Lambda_{7}$. The lighter color bands are the ones demanded by the group theoretical analysis but not relevant for the protection of the crossings. (D) Visual representation of the lower tilted Dirac cone that is circled in $2 \mathrm{~A}$. 
A

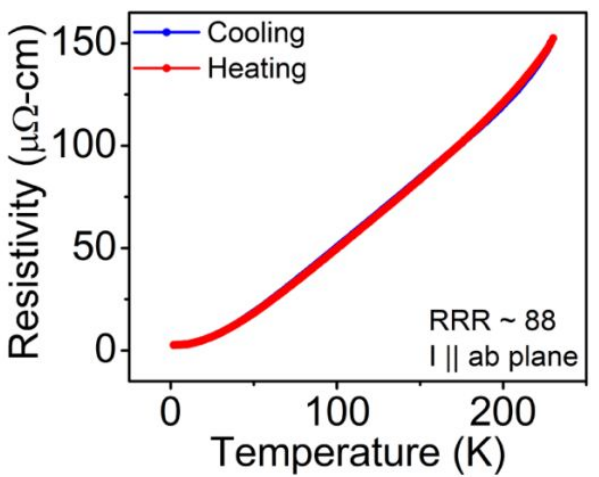

C

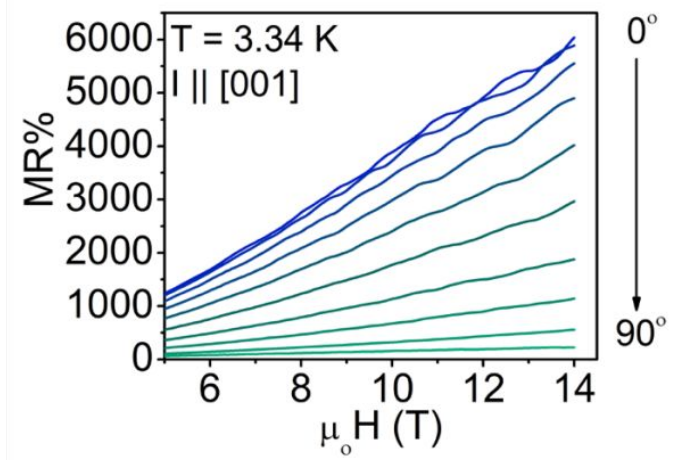

B
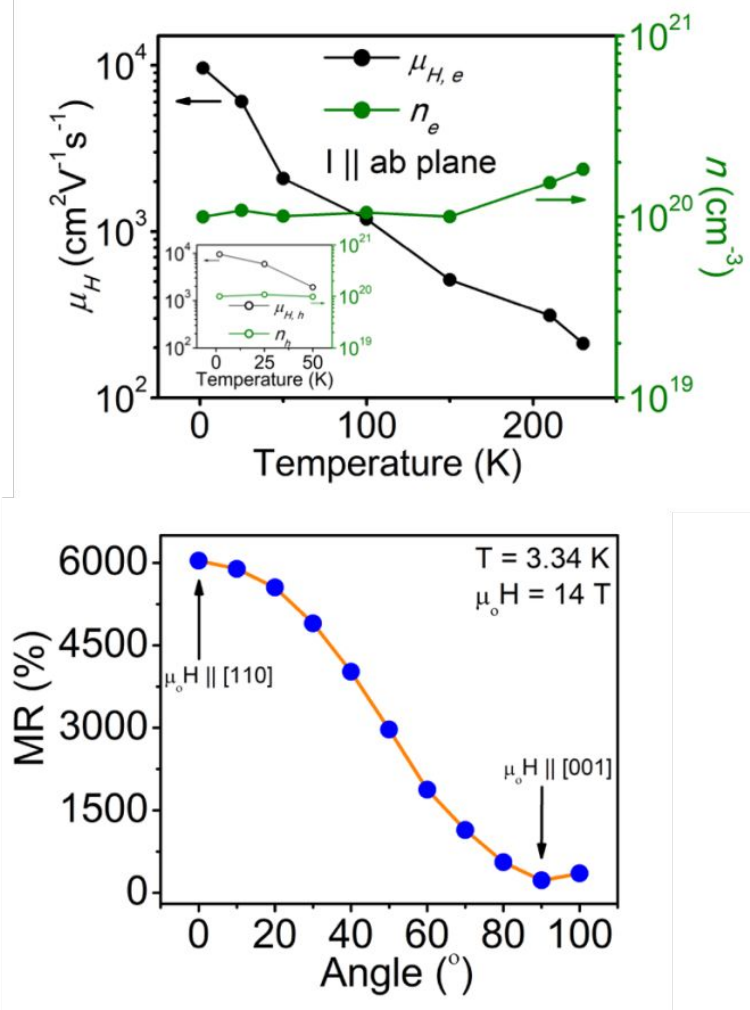

Figure 3: (A) Resistivity ( $\rho$ ) and (B) Hall transport data for $\operatorname{Ir}_{2} \operatorname{In}_{8} S$. $\operatorname{Ir}_{2} \operatorname{In}_{8} S$ has an electron and hole (inset) mobility of $\sim 10,000 \mathrm{~cm}^{2} \mathrm{~V}^{-1} \mathrm{~s}^{-1}$ at $1.8 \mathrm{~K}$ and a carrier concentration of $\sim 10^{20} \mathrm{~cm}^{-3}$. (C) Angle dependent magnetoresistance on an oriented crystal of $\operatorname{Ir}_{2} \operatorname{In}_{8} \mathrm{~S}$ at $3.34 \mathrm{~K}$ from 5 to $14 \mathrm{~T}$. (D) Maximum MR\% at $14 \mathrm{~T}$ as a function of angle; the approximately $\cos ^{2}(\theta)$ dependence of the magnetoresistance can be seen by the orange line. 

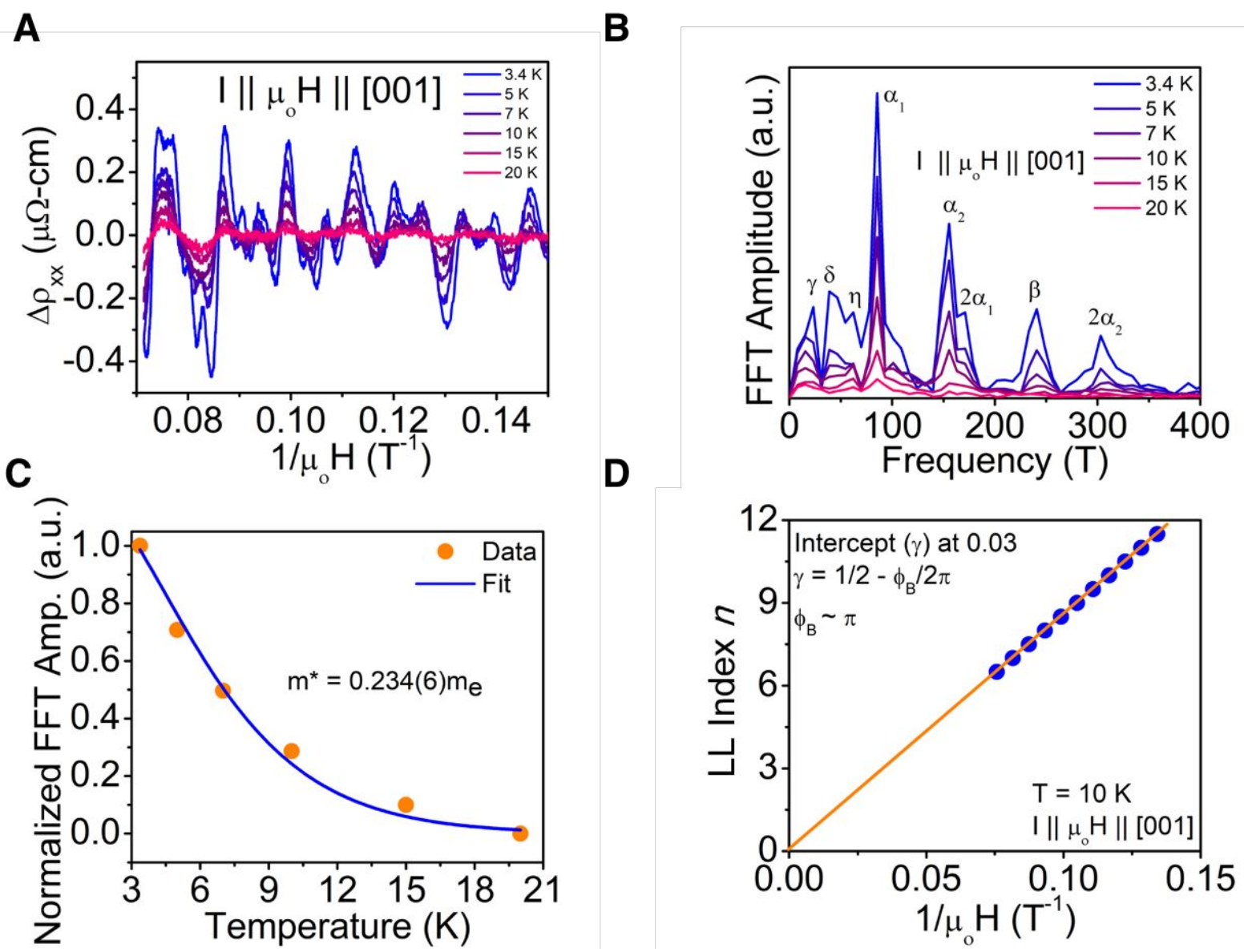

Figure 4: (A) Shubnikov de-Haas $(\mathrm{SdH})$ oscillations for $\operatorname{Ir}_{2} \mathrm{In}_{8} \mathrm{~S}$ as a function of temperature. The oscillations remain clearly visible up to $15 \mathrm{~K}$. (B) Fast Fourier Transform of the variable temperature $\mathrm{SdH}$ oscillations. Two predominant frequencies $\left(\mathrm{F}_{\alpha 1}\right.$ and $\left.\mathrm{F}_{\alpha 2}\right)$ can be seen at 86 and $155 \mathrm{~T}$, with another $\mathrm{F}_{\beta}$ pocket at $\sim 241 \mathrm{~T}$. (C) Lifshitz-Kosevich (LK) fit of the decay amplitude for the $\mathrm{F}_{\alpha 1}$ data, yielding effective mass $\mathrm{m}^{*}=0.234(6) \mathrm{m}_{\mathrm{e}}$. (D) Landau fan diagram from the $10 \mathrm{~K} \mathrm{SdH}$ data for $\mathrm{F}_{\alpha 1}$. 
For Table of Contents Only:
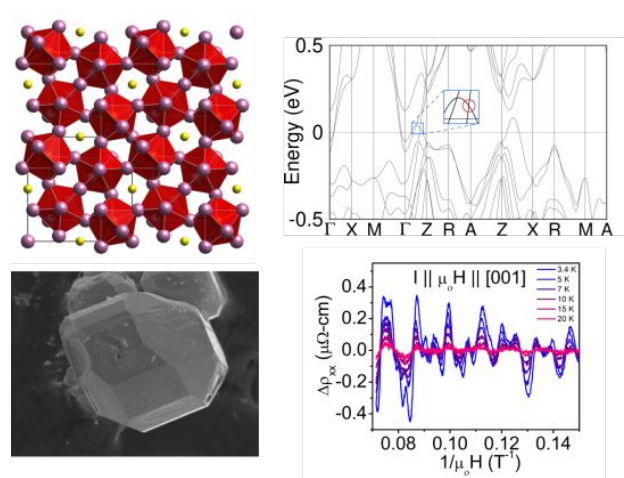